\title{
Epitaxial growth of ultrathin palladium films on $\operatorname{Re}\{0001\}$
}

Article

Accepted Version

Etman, H. A., Zheleva, Z. V., Held, G. and Bennett, R. A. (2011) Epitaxial growth of ultrathin palladium films on $\operatorname{Re}\{0001\}$. The Journal of Physical Chemistry C, 115 (10). pp. 4191-4199. ISSN 1932-7447 doi: https://doi.org/10.1021/jp112136f Available at https://centaur.reading.ac.uk/19500/

It is advisable to refer to the publisher's version if you intend to cite from the work. See Guidance on citing.

To link to this article DOI: http://dx.doi.org/10.1021/jp112136f

Publisher: American Chemical Society

All outputs in CentAUR are protected by Intellectual Property Rights law, including copyright law. Copyright and IPR is retained by the creators or other copyright holders. Terms and conditions for use of this material are defined in the End User Agreement.

\section{www.reading.ac.uk/centaur}

\section{CentAUR}

Central Archive at the University of Reading 
Reading's research outputs online 


\title{
The epitaxial growth of ultra-thin palladium films on
}

\section{$\operatorname{Re}\{0001\}$}

\author{
Haitham A. Etman, Zhasmina V. Zheleva, Georg Held and Roger A. Bennett ${ }^{a}$
}

School of Chemistry, University of Reading, Reading, RG6 6AD, UK

Ultra-thin bimetallic layers create unusual magnetic and surface chemical effects through the modification of electronic structure brought on by low dimensionality, polymorphism, reduced screening and epitaxial strain. Previous studies have related valence and core-level shifts to surface reactivity through the d-band model of Hammer and Nørskov, and in heteroepitaxial films this band position is determined by competing effects of coordination, strain and hybridization of substrate and overlayer states. In this study we employ the epitaxially matched $\operatorname{Pd}$ on $\operatorname{Re}\{0001\}$ system to grow films with no lateral strain. We use a recent advancement in low energy electron diffraction to expand the data range sufficiently for a reliable determination of the growth sequence and out-of-plane surface relaxation as a function of film thickness. The results are supported by scanning tunneling microscopy and x-ray photoemission spectroscopy, which show that the growth is layer-by-layer with significant core-level shifts due to changes in film structure, morphology and bonding.

\footnotetext{
${ }^{a}$ E-mail: r.a.bennett@reading.ac.uk, Telephone: +44 (0)118 3788559
} 
Keywords: Palladium, Rhenium, Structure, LEED-IV, thin film, Core-level shifts, STM, XPS

\section{Introduction}

Metallic thin films deposited on hexagonal single-metal crystals have received considerable attention in the past few decades ${ }^{1,2}$ due to the interest in modifying the chemical, electronic and magnetic properties of the surface in a systematic and controllable manner. Such metal overlayers and surface alloys lend themselves to a variety of unusual catalytic properties such as facile $\mathrm{H}_{2}$ dissociation and weak binding of $\mathrm{CO}$ which are beneficial in applications such as hydrogen fuel cells ${ }^{3}$. The most common substrate to be employed for the study of ultra-thin Pd films is the ruthenium $\{0001\}$ surface which has an in plane lattice constant of $2.71 \AA$. Pd layers grown on this surface are strained due to a lattice mismatch of circa $1.7 \%$ with respect to the $\operatorname{Pd}\{111\}$ in-plane spacing of $2.75 \AA$. In this study we employ the rhenium $\{0001\}$ surface as a substrate due to its very close epitaxial match of the in-plane lattice constant of $(2.76 \AA)$. With just $0.4 \%$ mismatch we expect epitaxial growth such that the surface defect density is low and as such the intrinsic properties of the Pd overlayer can be separated from variations induced by local perturbation of the lattice and strain. While the growth of $\mathrm{Pd}$ on $\mathrm{Ru}\{0001\}$ has recently been well characterized ${ }^{4}$ there is a lack of similar quantitative structural data for the $\mathrm{Pd}$ on $\operatorname{Re}\{0001\}$ system.

Campbell et al..$^{5}$ studied the deposition of $\operatorname{Pd}$ on $\operatorname{Re}\{0001\}$ and $\operatorname{Ru}\{0001\}$ from sub-monolayer (ML) to multilayers using X-ray photoelectron spectroscopy (XPS) and temperature programmed desorption (TPD) employing $\mathrm{CO}$ and $\mathrm{H}_{2}$ adsorption as probe molecules. They found a coverage dependent core level shift in the $\mathrm{Pd} 3 \mathrm{~d}_{5 / 2}$ peak position (decrease in the binding energy as coverage increases) when deposited on $\operatorname{Re}\{0001\}$. The peak shifts by $\sim 0.15 \mathrm{eV}$ over the range 0.2ML to $1 \mathrm{ML}$, after which a further continuous shift of $0.65 \mathrm{eV}$ was observed up to a coverage 
of $\sim 7 \mathrm{ML}$. Beyond $7 \mathrm{ML}$, no change in the peak position was observed up to 40ML Pd, the thickest films studied. TPD and XPS experiments on the adsorption of CO on $1 \mathrm{ML}$ of Pd on $\operatorname{Re}\{0001\}$ revealed that the desorption temperature of $\mathrm{CO}$ from the system is $\sim 110 \mathrm{~K}$ lower than that from $\mathrm{Pd}(100)$, indicating that Pd-Re interactions dramatically weakens the Pd-CO bond strength. These results were attributed to a charge transfer from the Pd adlayers to the $\operatorname{Re}$ substrate. Pick confirmed these results using self-consistent tight binding calculations of CO chemisorption on monolayer Pd deposited on $\operatorname{Re}\{0001\}$ and $\operatorname{Ru}\{0001\}^{6}$. Very recently Golfetto et al. ${ }^{7}$ employed high resolution XPS to resolve Pd $3 \mathrm{~d}_{5 / 2}$ core level shifts associated with the clean surface and $\mathrm{Pd}$ films on $\mathrm{Ru}\{0001\}$. The results identified layer specific core level shifts which could be used as an experimental descriptor of chemical reactivity.

Several theoretical studies using density functional theory (DFT) have been performed on the 1 ML Pd on $\operatorname{Re}\{0001\}$ system. Wu and Freeman ${ }^{8}$ have compared the bonding mechanism of Pd monolayers on various transition metals and showed that interfacial dipoles (i.e. charge polarisation induced in the Pd layer by the substrate) play a key role in the bonding mechanism. Charge accumulates in the interfacial region on top of the Re and is depleted from both $\operatorname{Re}$ and Pd atoms, which lowers the energies of the Pd states. They predict the hcp site to be preferred for 1 ML Pd on $\operatorname{Re}\{0001\}$. Pallassana et al. ${ }^{9}$ studied the chemisorption of hydrogen on the same surface and based their interpretation of the results on the d-band model developed by Hammer and Nørskov ${ }^{10}$. They related the weak chemisorption of hydrogen on 1 ML Pd on $\operatorname{Re}\{0001\}$ surface to a substantial lowering in the valence d-band centre of Pd by $0.72 \mathrm{eV}$ below that of the bulk Pd. This lowering was later confirmed experimentally by Mun et al. ${ }^{2}$ who further suggested that the growth mode is of the Stranski-Krastanov type because of the manner in which the Pd $3 \mathrm{~d}_{5 / 2}$ core level shifts and the peak shape changes as a function of coverage. 
The rigid d-band model of Ruban et al. ${ }^{11}$ predicts the d-band shifts in heteroepitaxial and surface alloy systems. The model uses changes in electron embedding density inferred from the elemental Wigner-Seitz radius and the local co-ordination of the substrate atom pair to predict core level shifts. While the model predicts the trend for $\operatorname{Pd}$ on $\operatorname{Ru}\{0001\}$, for $\operatorname{Pd}$ on $\operatorname{Re}\{0001\}$ the Wigner-Seitz radius of both elements is identical and only a very small shift would be expected for unrelaxed surfaces ${ }^{11}$. In general, under-coordinated surface, step and kink atoms on transition metal surfaces $\left(\mathrm{Pd}^{7,12}, \mathrm{Pt}^{13}, \mathrm{Rh}^{14}\right)$ consistently show lower binding energies with decreasing co-ordination, i.e. the opposite shift to the established experimental data for $\mathrm{Pd}$ on $\operatorname{Re}\{0001\}$.

The physics of the modifications to the valence band structure of Pd to induce ferromagnetic order have lead to much interest in potential magnetic properties. A slight increase in the local density of states at the Fermi level is required to fulfill the Stoner criterion for ferromagnetism which may be brought about by growing strained layers, alternate polymorphs or nanoparticles ${ }^{15}$. In a DFT study of various Pd polymorphs, Hüger and Osuch ${ }^{16}$ identified the onset of ferromagnetism with lattice expansion and showed thresholds at $\sim 10 \%$ lattice expansion for fcc Pd, but that hcp and double-hcp (dhcp) are ferromagnetic at the optimum bulk lattice spacing (which was identical for all polymorphs). Hüger and Osuch also experimentally attempted to grow hcp structures on W(100) and reported flat bands in angle-resolved UPS near the Fermi level implying that Pd becomes ferromagnetic. More recent DFT calculations by Alexandre et $a l .{ }^{17}$ predicted that bulk Pd in hcp structures to be ferromagnetic and the fcc phase paramagnetic. However, two dimensional defects, such as surfaces, twin boundaries and stacking faults with locally hcp stacking in an fcc lattice increased the magnetic susceptibility. The (111) surface is paramagnetic, but has a large susceptibility, and was suggested to have a propensity to become 
magnetic if in close proximity to stacking faults. The stacking sequence and structure is clearly of relevance to a detailed understanding of magnetism in Pd thin films.

No experimental structural analysis of $\operatorname{Pd} / \operatorname{Re}\{0001\}$ has been published (in contrast $\mathrm{Pd} / \mathrm{Ru}\{0001\}$ has been studied by de Siervo et al. ${ }^{18}$ and later by Santos et $\left.a l .{ }^{4}\right)$. In this study we present the first detailed structural analysis of ultra-thin $\operatorname{Pd}$ films on $\operatorname{Re}\{0001\}$ in the range 0 - 4 ML investigated by a combination of quantitative LEED, scanning tunneling microscopy (STM) and XPS. We show that Pd grows epitaxially with the first layer following the hep sequence of the substrate and subsequent layers growing in fcc structure. The use of non-normal incidence data in LEED structural analysis beams allows a far greater effective range of useful energies to be considered.

\section{Experimental Procedures and Structure Analysis}

\subsection{Experiment}

The experiments were undertaken in a twin UHV chamber system described in detail previously ${ }^{19,20}$ which has now been modified to incorporate a small scanning tunneling microscope operating at room temperature with an associated sample transfer system. The preparation chamber is equipped with a Vacuum Generators 3 grid low energy electron diffraction (LEED) system with a 16 bit peltier cooled ccd camera to capture LEED images and an Oxford Applied Research water-cooled e-beam evaporator for Pd deposition. XPS was performed in a mu-metal analyser chamber equipped with a hemispherical analyser and an unmonochromated $\mathrm{Al} \mathrm{K} \alpha \mathrm{X}$-ray source $(\mathrm{h} v=1486.6 \mathrm{eV})$. Binding energies are referenced to the $\operatorname{Re} 4 \mathrm{f} 7 / 2$ at $40.2 \mathrm{eV}$ with positions assigned on the basis of mixed Gaussian-Lorentzian product peakshape on a Shirley background. The homemade STM is mounted in a six-way cross on the analyser chamber and operates in UHV at room temperature with mechanically cut 
platinum/iridium (Pt80/Ir20) tips. The STM is controlled by an Oxford Instruments TOPS III system. Lateral length scales were calibrated by atomic resolution imaging of a c $(6 \times 2)$ oxide superstructure on $\mathrm{Cu}\{110\}$ resulting in a largest scan size of $8000 \AA$. The $\mathrm{z}$-scale is calibrated from the images of the clean Re surface reported here. Single crystals are held in place by Ta sheet or $\mathrm{W}$ wire inserted into a narrow slit around the crystal perimeter and spotwelded to tantalum sample plates. These plates are transferred from the STM to the main analysis chamber transfer arm for e-beam heating, cleaning, deposition, XPS and LEED analysis. Sample temperatures were initially calibrated and measured by a c-type thermocouple spot welded on the side of the crystal but the thermocouple was later removed to aid sample transfer. Temperatures were subsequently inferred from measurements of heating power employed and the previous calibration data.

The sample is cleaned by repeated cycles of flash heating to $\sim 2300 \mathrm{~K}$, followed by a short anneal at $\sim 1300 \mathrm{~K}$ in $5 \times 10^{-8}$ Torr $\mathrm{O}_{2}$ for $\sim 20$ minutes to remove carbon, the initial primary contaminant, and then flashed to desorb oxygen. This cycle was repeated until the LEED pattern indicated a well-ordered $(1 \times 1)$ hexagonal surface and the XPS showed a clean surface. To remove all oxygen two final flashes were found to be necessary. Pd was deposited at $\sim 0.7$ $\mathrm{ML} / \mathrm{min}$ onto the Re surface as it cooled to RT (typically about $373 \mathrm{~K}$ ) with no post deposition anneal. The Pd flux was monitored using the ion current meter integral to the source (typically $\sim 12 \mathrm{nA}$ ) which also takes a high fraction of the charged $\mathrm{Pd}$ from the beam (reducing the possibility that these influence the growth mode significantly). The flux was calibrated using the photoelectron intensity of the $\mathrm{Pd} 3 \mathrm{~d}$ peaks (measured at $50 \mathrm{eV}$ pass energy) as a function of time to identify monolayer break points. 
LEED-IV experiments were performed on the clean surface using three angles of incidence (polar angles of $+3^{\circ},-7^{\circ}$ and $+10^{\circ}$ ) in the energy range of $75-300 \mathrm{eV}$ and on the $1,2,3$ and 4 ML Pd films using two angles incidence (normal and $10^{\circ}$ off-normal) in the energy range of $50-$ $255 \mathrm{eV}$. At normal incidence, all the spots of the same order are equivalent and were therefore averaged $^{21}$ (i.e. 3 sets of spots are being used for normal incidence $(1,0),(1,1)$ and $(2,0)$ plus 18 for the off-normal data with the calculations run simultaneously). The LEED patterns were all of high intensity with a low background and did not contain any superstructure spots. Stray light from the electron gun was background subtracted from each 16-bit image. The total energy ranges for the analysis were $7040 \mathrm{eV}$ for the clean surface and between 1670 and $1900 \mathrm{eV}$ for each of the films.

\subsection{LEED-IV Calculations}

The program used to compare the theoretical and experimental intensity versus electron energy (IV) curves is CLEED ${ }^{22}$. The LEED-IV calculations ${ }^{23}$ are based on fully dynamical scattering theory described by Pendry ${ }^{24}$ and Van Hove and Tong ${ }^{25}$. Technical details and a full set of resulting data are given in Supplementary Information $^{26}$. The agreement between the experimental and theoretical IV curves was quantified with Pendry's $R_{P}$ factor and the error limits for the determined parameters were calculated using the RR factor method ${ }^{27}$. In all calculations a $(1 \times 1)$ periodicity was assumed. The angle of incidence was optimized as part of the structural optimization, which increases the number of search parameters by 2 .

A recent modification to the program allows for the simultaneous fitting of multiple data sets acquired from the same sample at different angles of incidence. The breaking of symmetry, in comparison to experiments with carefully aligned normal incidence, allows an effective increase of the energy range as LEED spots are no longer symmetrically equivalent. Thus more complex 
structural models can be addressed; in this case surface structures with inequivalent domains mixing spot intensities.

\section{Results and discussion}

\subsection{Clean $\operatorname{Re}\{0001\}$ structure}

The $\operatorname{Re}\{0001\}$ surface is the most stable truncation of the crystal and exhibits large terraces. STM images of the clean surface, Figure 1, show single steps separating terraces of width ranging from 150 to $860 \AA$. The average single step size was used to calibrate the $\mathrm{z}$-scale of the STM to be consistent with the interlayer spacing for the $\operatorname{Re}\{0001\}$ surface $\sim 2.3 \AA$ within small error margins. The step edges are extremely well defined and straight over extended distances. We did not achieve atomic resolution on the clean surfaces so cannot be explicit in assigning the direction of the steps but we expect them to run in the close-packed directions .

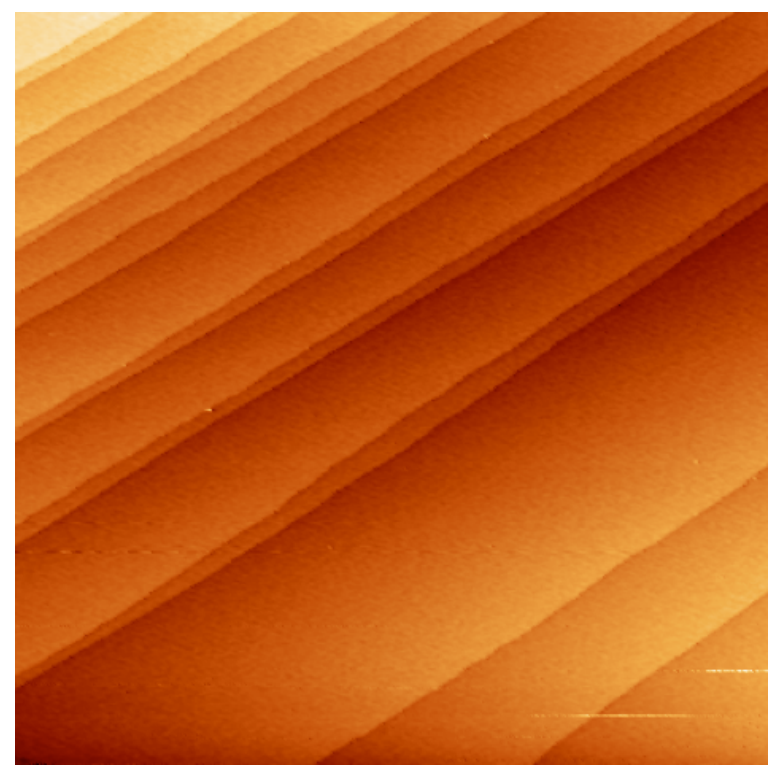

Figure 1. $(3000 \AA \times 3000 \AA)$ STM image of clean $\operatorname{Re}\{0001\}$ surface taken at 0.1 nA tunelling current and $1 \mathrm{~V}$ bias voltage showing wide terraces and straight monatomic step edges.

An ideal step free $\{0001\}$ basal plane of an hexagonal close packed surface has threefold symmetry, however, on examining the surface using conventional LEED, a sixfold symmetric 
pattern is observed at normal incidence. Figure 2 explains how this pattern is generated by the presence of monatomic steps at the surface which introduce two types of terraces, A-terminated and B-terminated (where A and B represent the two possible terminations of the hcp stacking sequence). The diffraction pattern from both terrace types is three-fold symmetric but each is a mirror image (or rotated by $180^{\circ}$ ) ${ }^{28}$ with respect to the other, resulting in each LEED spot being an incoherent sum of diffraction intensities from both terraces.

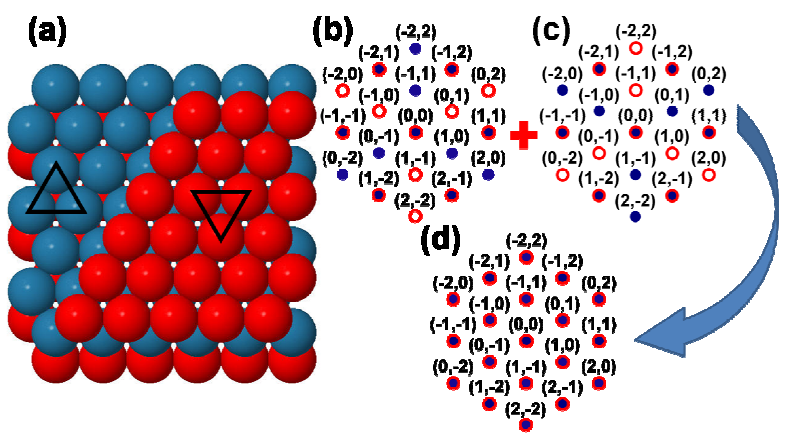

Figure 2. (a) hard sphere model of the hcp $\{0001\}$ surface with a monoatomic step between an A- and B-terminated terrace; different colours represent atoms in A and B layers. The triangles surrounding hcp hollow sites (above a second layer atom) indicate that the terraces are mirror images of each other. (b) and (c) schematic LEED patterns of the A- and B-terminated terraces; filled and open circles represent spots related by the surface symmetry. Symmetry-related spots of the same order (distance from the specular $(0,0)$ spot) have the same LEED-IV curves if data are taken at normal incidence. (d) Superposition of the LEED patterns from both terraces representing the six-fold symmetry observed in the experiment at normal incidence.

For the $\{0001\}$ basal plane the terraces are degenerate in energy and are expected to occur with equal abundance at thermodynamic equilibrium on the surface. The LEED-IV data are therefore an average over the two terrace types. Figure $2 b-d$ show how diffraction patterns 
generated by each terrace (filled and open circles) add to give a 6-fold symmetric LEED pattern at normal incidence. In Figure 3 we show sample LEED-IV data taken at $3^{\circ}$ off normal and at larger angles $\left(-7^{\circ}\right.$ and $\left.+10^{\circ}\right)$ from the clean surface. The left and right panels show the results for $(-1,0)$ and $(0,-1)$ first order LEED spots, respectively. At $3^{\circ}$ off normal incidence they are very similar (but not identical), however, for increasing angles away from normal incidence large differences emerge. This is because the symmetry of the diffraction geometry is broken at offnormal incidence and features from different terraces become inequivalent. Some of the terraces shown in Figure 1 are separated by closely bunched steps and some of these terraces are therefore relatively small leading to a local bias in step termination (more A terrace than B). However, this is a very small sample area in comparison to that measured by conventional LEED and we expect local biases on small lengthscales in step termination to cancel out, and indeed later STM images of Pd coated surfaces bear this out.

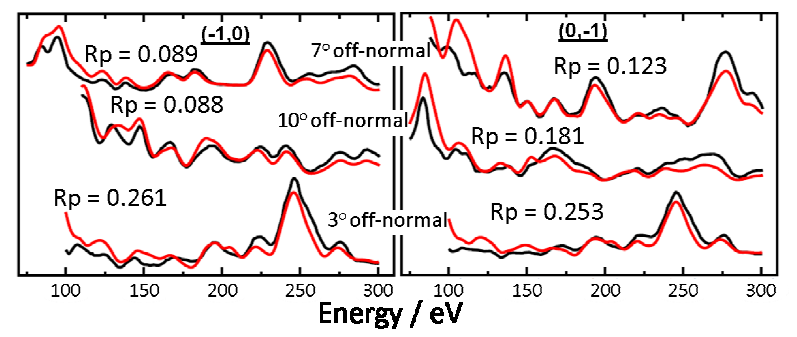

Figure 3. Sample LEED-IV curves for two spots representing the two types of terraces, $(-1,0)$ on the left and $(0,-1)$ on the right, with Pendry's $\mathrm{R}$-factors $\left(\mathrm{R}_{\mathrm{P}}\right)$. Black lines represent experimental curves and red lines represent theoretical fits. At $3^{\circ}$ incidence both $(-1,0)$ and $(0,-1)$ have similar structure. Further off normal the curves for each spot look dissimilar as the symmetry is broken and the curves contain independent information.

Following from the above model, the surface structure of the inter-layer spacing of the top four atomic layers was determined by simultaneously fitting models for the three angles with the 
assumption that there was no dominant terrace type on the surface (i.e. a 1:1 mix of A and B terminations). The best $\mathrm{R}_{\mathrm{P}}$-factor obtained is 0.155 for a relaxed structure of the clean surface, a summary of the results is shown in Table 1. The pattern of surface relaxation (a top layer contraction, second layer slight expansion and third layer small contraction) is common to most metal surfaces ${ }^{29}$. The experimental results are smaller in size, however, than the large relaxations predicted theoretically for the outermost atoms of this surface ${ }^{8,9}$.

Table 1. Summary of LEED-IV results obtained for the clean surface

\begin{tabular}{|c|c|c|c|c|c|}
\hline Polar Angle & $3.11 \pm 0.12^{\circ}$ & \multicolumn{2}{|c|}{$9.93 \pm 0.05^{\circ}$} & \multicolumn{2}{|c|}{$6.94 \pm 0.09^{\circ}$} \\
\hline Azimuthal Angle & $176.06 \pm 0.06^{\circ}$ & \multicolumn{2}{|c|}{$102.81 \pm 0.08^{\circ}$} & \multicolumn{2}{|c|}{$238.98 \pm 0.1^{\circ}$} \\
\hline $\mathrm{R}_{\mathrm{P}}$ & 0.158 & \multicolumn{2}{|l|}{0.132} & \multicolumn{2}{|c|}{0.167} \\
\hline $\mathrm{RR}$ & 0.112 & \multicolumn{2}{|l|}{0.117} & \multicolumn{2}{|c|}{0.123} \\
\hline Energy Range / eV & 2573.0 & \multicolumn{2}{|c|}{2341.25} & \multicolumn{2}{|c|}{2126.25} \\
\hline Average $R_{P}$ & \multicolumn{5}{|l|}{0.152} \\
\hline Average RR & \multicolumn{5}{|l|}{0.067} \\
\hline Total Energy Range / eV & \multicolumn{5}{|l|}{7040.5} \\
\hline Interlayer separation & \multicolumn{2}{|l|}{ Experiment } & \multicolumn{3}{|l|}{ Theory } \\
\hline $\mathrm{d}_{12} / \AA$ & \multicolumn{2}{|c|}{$2.13 \pm 0.02(-4.3 \pm 0.8 \%)$} & \multicolumn{2}{|c|}{$2.07(-7.4 \%)$} & $2.06(-7.6 \%)$ \\
\hline $\mathrm{d}_{23} / \AA$ & \multicolumn{2}{|c|}{$2.28 \pm 0.02(+2.3 \pm 0.8 \%)$} & \multicolumn{2}{|c|}{$2.32(+3.9 \%)$} & $2.28(+2.3 \%)$ \\
\hline $\mathrm{d}_{34} / \AA$ & \multicolumn{2}{|c|}{$2.19 \pm 0.02(-1.7 \pm 1 \%)$} & \multicolumn{2}{|l|}{ bulk } & - \\
\hline$\overline{\mathrm{d}_{45} / \AA}$ & \multicolumn{2}{|c|}{$2.20 \pm 0.03(+1.3 \pm 1.5 \%)$} & \multicolumn{2}{|c|}{ From ref [6] } & From ref [8] \\
\hline $\mathrm{d}_{\mathrm{bulk}} / \AA$ & \multicolumn{5}{|l|}{2.23} \\
\hline
\end{tabular}

\subsection{Ultra-thin Pd Films}

\subsubsection{XPS and Core-level shifts}


The Pd $3 \mathrm{~d}$ photoemission was monitored as a function of deposition time to establish a calibration curve for the evaporator flux as shown in Figure 4. Figure 4 a shows the $\mathrm{Pd} 3 \mathrm{~d}_{5 / 2}$ region for a succession of different coverages, while Figure $4 \mathrm{~b}$ shows the aggregated intensity of the entire Pd $3 d$ photoemission. The clear break points in the Figure $4 \mathrm{~b}$ intensity vs. flux curves immediately suggest a layer by layer growth mode for at least the first two monolayers, as expected for an epitaxial system with very little strain. We assign the breaks at 780 nA.s and 1600 nA.s to the completion of the first and second monolayers, respectively.

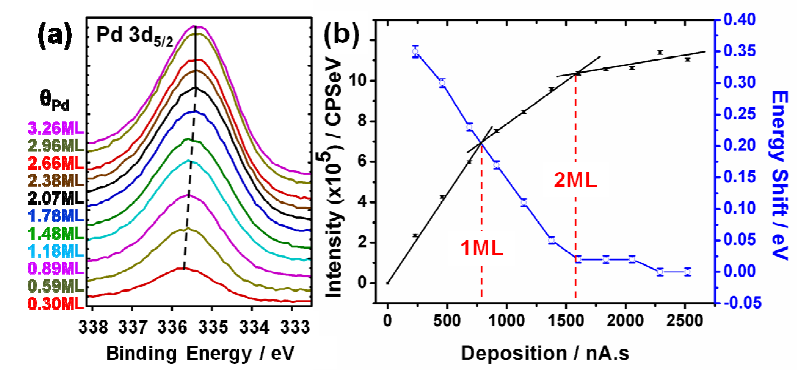

Figure 4. Core-level shifts for Pd $3 d_{5 / 2}$ XPS signal (a) XPS signal of various coverages with black dotted line showing shift in peak position and the solid one showing no shift for higher coverage (b) Calibration of the coverage by monolayer break point analysis of the intensity of Pd $3 \mathrm{~d}$ signal versus deposition time multiplied by evaporator ion flux and a plot of the $\mathrm{Pd} 3 \mathrm{~d}_{5 / 2}$ core level shift (from $335.69 \mathrm{eV}$ to $335.34 \mathrm{eV}$ ) as a function of increasing Pd deposition.

While synchrotron radiation studies have managed to resolve the $\mathrm{Pd} 3 \mathrm{~d}_{5 / 2}$ peak into multiple components $^{1}$, here we work with an unmonochromated X-ray source and we resolve one broad peak which shifts systematically as a function of layer thickness. In figure $4 \mathrm{~b}$ we show a continuous shift in binding energy from $335.69 \mathrm{eV}$ at approximately $0.30 \mathrm{ML}$ to $335.36 \mathrm{eV}$ at the completion of $2 \mathrm{ML}$ and then a very slow and small change to $335.34 \mathrm{eV}$ over the next monolayer. The uncertainties in the core-level shifts were determined using the in-built Monte Carlo 
simulation function in CasaXPS ${ }^{30}$ and were found to be of the order of a few $\mathrm{mV}$ (including uncertainties in the $\operatorname{Re} 4 \mathrm{f}_{7 / 2}$ reference energy assignments). Previous work by Goodman shows a similar change in binding energy over a larger range of coverage (in that work the coverage was determined calibrating to an assumed Pd ML temperature programmed desorption peak and not directly as here). ${ }^{5}$

\subsubsection{Scanning Tunneling Microscopy}

The deposition of Pd films was studied using STM from submonolayers to just above 1ML. The STM images, Figure 5 support the interpretation of the growth mode to be layer by layer. For submonolayer deposition of $\sim 0.25 \mathrm{ML}$ Pd onto a room temperature substrate the Pd forms fractal islands on the terraces which do not appear to preferentially wet the step edges as shown in Figure 5a. Similar islands have been observed before in homo and heteroepitaxial growth, and form due to diffusion-limited aggregation ${ }^{31,32}$. At higher coverage, as shown in Figure $5 b$, these islands connect and fill in to form more compact islands losing their fractal shapes as the fractal "branches" thicken. They do not, however, form compact islands as the island peripheries remain strongly structured with a lack of straight steps leading down to the Re terrace. The islands are of a single Pd layer height (i.e. they wet the surface rather than growing as multilayered islands). The Pd islands contact the straight Re step edges as they grow, however the step edge is still distinguishable as a straight feature between the islands. At the completion of $1 \mathrm{ML}$, almost the whole surface is covered with Pd leaving just a small part of the surface not covered, which is a normal phenomena observed even in homoepitaxial growth of metals. In Figure $5 \mathrm{c}$ and $5 \mathrm{~d}$, a $1.25 \mathrm{ML}$ Pd film was grown and we can immediately see that most of the surface is covered before the second layer starts to grow ( $\sim 7 \%$ of the Re surface is not covered). The film tends to extend across the terraces but stops at the step-edges and so reproduces the 
underlying Re straight step structure. However, it is apparent that regions growing alongside the small amount of uncovered Re still maintain the heavily structured Pd down to Re step geometry as seen in Figure $5 b$. Pd growing as a second layer on the film forms compact islands with approximately hexagonal structure as expected for (111) oriented structures. There is little tendency to connect directly to the neighbouring step edges and most second layer Pd appears in the middle of the terraces. Of the islands which do appear to be growing from the step edges onto the terrace a minority appear to be continuous and most of them have a grain boundary or discontinuity at the interface. 

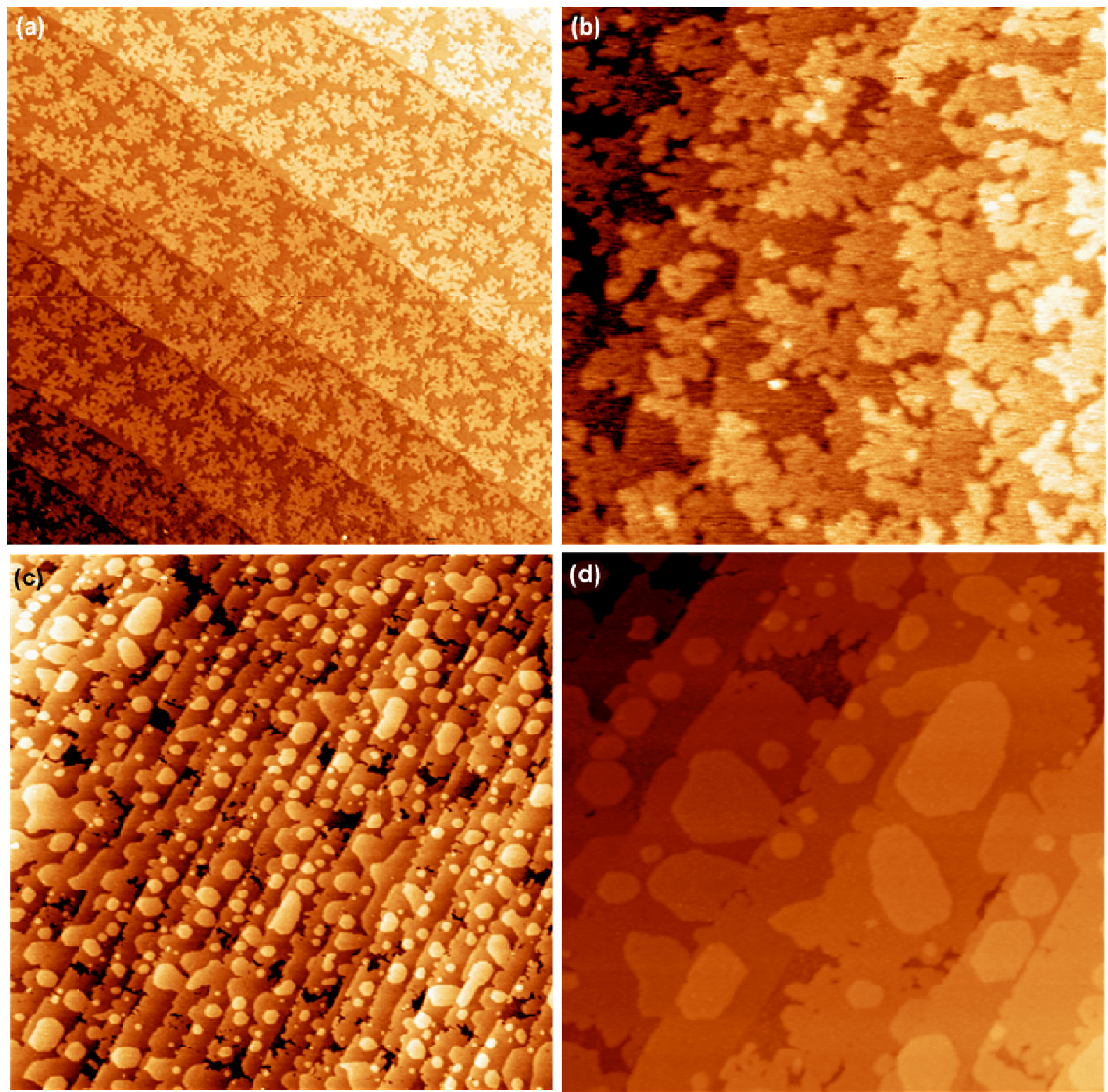

Figure 5. STM images of multiple Pd coverage on the surface: (a) $(2700 \AA \times 2700 \AA)$ image taken using $0.1 \mathrm{nA}$ tunneling current and $2 \mathrm{~V}$ bias voltage of $\sim 0.25 \mathrm{ML}$ fractal islands that do not wet the step edges. (b) $(1130 \AA \times 1130 \AA)$ image taken using $0.1 \mathrm{n} A$ and $-1 \mathrm{~V}$ of $\sim 0.4 \mathrm{ML}$ compact islands of Pd with no visible straight step edges. (c) $(4440 \AA \times 4440 \AA)$ image taken using $0.5 n A$ and $2 \mathrm{~V}$ of $\sim 1.25 \mathrm{ML} \mathrm{Pd}(\mathrm{d})(1500 \AA \times 1500 \AA)$ taken using $0.5 \mathrm{nA}$ and $2 \mathrm{~V}$ bias voltage of 1.25ML Pd layers. 
In Figure 6 we show a closer examination of the islands and films on the terraces along with line profiles across features of interest. We can also report that the STM imaging reveals a distinction between Pd monolayer and the Re substrate on the next terrace up (i.e. in the same crystal plane); the Pd tends to image with an apparent height higher than the neighbouring $\operatorname{Re}$ due to a chemical contrast. We did not find, however, that this contrast was particularly sensitive to the tunneling conditions employed. In Table 2 we present the measured apparent heights from the STM images along the same profile taken across three images of the same area. The clean Re-Re step height images as $\sim 2.2 \AA$ for most images as expected as this measurement should not show any chemical contrast. The 1 ML Pd apparent height is measured from Re substrate to the edge of the film and has a chemical difference between $\mathrm{Re}$ and Pd and a small contrast difference with tunnel current. The 2 ML Pd height is the apparent height of an island on a 1 ML Pd film on a terrace and may have contrast differences in the electronic structure of 1 and 2 ML thick films. The final contrast occurs where a Pd film on a lower terrace meets a clean Re upper terrace (i.e. the terrace switches from Re to Pd termination), in which case the Re appears at significantly lower apparent height than the Pd.

Table 2. Dependence of apparent height of surface structures on tunneling condition.

\begin{tabular}{|c|c|c|c|}
\hline Sample Bias / V & +2.0 & -2.0 & +2.0 \\
\hline Tunnel current / nA & 1.0 & 1.0 & 4.0 \\
\hline $\operatorname{Re}-\operatorname{Re}$ step / $\AA$ & 1.98 & 2.17 & 2.17 \\
\hline $1 \mathrm{ML} \mathrm{Pd}-\operatorname{Re} / \AA$ & 2.71 & 2.25 & 2.19 \\
\hline 2ML Pd - 1ML Pd / & 2.10 & 2.32 & 2.04 \\
\hline $1 \mathrm{ML}$ Pd - Re in plane / $\AA$ & 0.80 & 0.66 & 0.68 \\
\hline
\end{tabular}




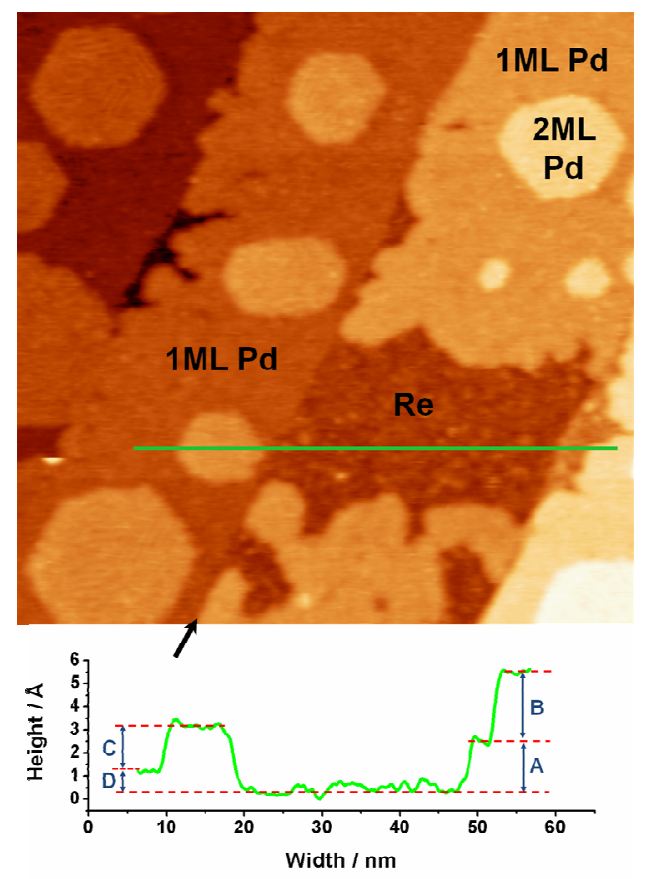

Figure 6. $(640 \AA \times 640 \AA)$ STM image of $1.25 \mathrm{ML}$ Pd deposited on the surface. The arrow points along the straight step edge of the substrate. The line profile shows the heights of the different features on the surface: (A) is the Re - Re step height, (B) 1ML Pd - Re, (C) 2ML Pd - 1ML Pd and (D) $1 \mathrm{ML}$ Pd - Re in plane. The values are given in Table 2.

\subsubsection{LEED-IV analysis}

Pd was deposited at just above room temperature (sample had cooled from the flash desorption of oxygen to $<373 \mathrm{~K}$ ) to form ultra-thin films of thickness of 1, 2, 3 and $4 \mathrm{ML}$. Each layer was examined using LEED-IV. The pattern observed was a $(1 \times 1)$ hexagonal pattern qualitatively the same as the substrate, suggesting a pseudomorphic growth. Since the LEED images showed a hexagonal pattern and the lattice mismatch between $\mathrm{Pd}$ and $\mathrm{Re}$ is negligible $(\sim 0.4 \%)$, a hollow site would be the most obvious adsorption site for the first layer of Pd atoms. These may adopt an hcp stacking sequence (adatom above a second layer substrate atom) or an fcc structure (adatom above an empty substrate site in the second layer). Subsequent layers may introduce 
stacking faults or continue to grow as substrate layers (or could potentially re-arrange the previous layer). Thus multiple layer stacking leads to a plethora of different models. A comprehensive trial of possible models was undertaken for each film, including differing film thicknesses to probe our sensitivity to systematic errors in coverage calibration. Table 3 shows the models with their resulting $R_{P}$ and $R R$ factors. The lowest $R_{P}$ factors are all below those reported for atomic adsorbates, such as $\mathrm{S}$ on $\operatorname{Re}\{0001\}^{33}$. Competing models with similar $\mathrm{R}_{\mathrm{P}}$ can be distinguished by evaluating the upper limit $R_{P \max }=(1+R R) \times R_{P \min }$ (where $R_{P \min }$ is the lowest $\mathrm{R}$-factor). If a trial model has an $\mathrm{R}_{\mathrm{P}}$ factor higher than $\mathrm{R}_{\mathrm{Pmax}}$ then it is statistically unlikely and can be ignored. The $\mathrm{R}_{\mathrm{P}}$ factors within the statistical error margin of $\mathrm{R}_{\mathrm{Pmin}}$ are printed in bold characters. 
Table 3. Summary of attempted models with their associated $R_{P}$ and RR factors ${ }^{b}$

\begin{tabular}{|c|c|c|c|c|c|c|c|}
\hline $\begin{array}{c}\text { No. of } \\
\text { ML }\end{array}$ & $\begin{array}{l}\text { Stacking } \\
\text { Sequence }\end{array}$ & $\mathrm{R}_{\mathrm{P}}$ & RR & $\begin{array}{c}\text { No. of } \\
\text { ML }\end{array}$ & $\begin{array}{l}\text { Stacking } \\
\text { Sequence }\end{array}$ & $\mathrm{R}_{\mathrm{P}}$ & $\mathrm{RR}$ \\
\hline \multirow{7}{*}{1} & $\overline{\mathrm{ABABA}}$ & $\begin{array}{c}0.574 \\
\end{array}$ & 0.112 & \multirow{17}{*}{3} & $\overline{\mathrm{ABAB} c b c}$ & 0.420 & 0.133 \\
\hline & $\underline{\mathbf{A B A B}} a$ & $\underline{0.218}$ & $\underline{0.112}$ & & $\mathrm{ABAB} a b a b$ & 0.412 & 0.133 \\
\hline & $\mathrm{ABAB} c$ & 0.465 & 0.112 & & $\mathrm{ABAB} a b a c$ & 0.332 & 0.133 \\
\hline & $\mathrm{ABAB} a b$ & 0.462 & 0.112 & & $\mathbf{A B A B} a b c a$ & 0.272 & 0.133 \\
\hline & $\mathrm{ABAB} a c$ & 0.562 & 0.112 & & $\mathrm{ABAB} a b c b$ & 0.408 & 0.133 \\
\hline & $\mathrm{ABAB} c a$ & 0.573 & 0.112 & & $\mathrm{ABAB} a c a b$ & 0.343 & 0.134 \\
\hline & $\mathrm{ABAB} c b$ & 0.510 & 0.112 & & $\mathrm{ABAB} a c a c$ & 0.408 & 0.133 \\
\hline \multirow{12}{*}{2} & $\overline{\mathrm{ABAB} a}$ & 0.577 & 0.121 & & $\mathrm{ABAB} a c b a$ & 0.310 & 0.134 \\
\hline & $\mathrm{ABAB} c$ & 0.561 & 0.121 & & $\mathrm{ABAB} a c b c$ & 0.402 & 0.133 \\
\hline & $\mathrm{ABAB} a b$ & 0.351 & 0.121 & & $\mathrm{ABAB} c a b a$ & 0.406 & 0.133 \\
\hline & $\underline{\mathbf{A B A B}} a c$ & $\underline{0.211}$ & $\underline{0.121}$ & & $\mathrm{ABAB} c a b c$ & 0.312 & 0.134 \\
\hline & $\mathrm{ABAB} c a$ & 0.268 & 0.121 & & $\mathrm{ABAB} c a c a$ & 0.410 & 0.133 \\
\hline & $\mathrm{ABAB} c b$ & 0.386 & 0.121 & & $\mathrm{ABAB} c a c b$ & 0.349 & 0.133 \\
\hline & & 0.380 & 0.121 & & $\mathrm{ABAB} c b a b$ & 0.410 & 0.133 \\
\hline & $\mathrm{ABAB} a b c$ & 0.324 & 0.121 & & $\mathbf{A B} \mathbf{A B} c b a c$ & 0.270 & 0.133 \\
\hline & $\mathrm{ABAB} a c a$ & 0.410 & 0.121 & & $\mathrm{ABAB} c b c a$ & 0.343 & 0.133 \\
\hline & $\mathrm{ABAB} a c b$ & 0.353 & 0.121 & & $\mathrm{ABAB} c b c b$ & 0.420 & 0.133 \\
\hline & $\mathrm{ABAB} c a b$ & 0.366 & 0.121 & \multirow{2}{*}{4} & $\mathrm{ABAB} a c a$ & 0.464 & 0.127 \\
\hline & $\mathrm{ABAB} c a c$ & 0.425 & 0.121 & & $\mathrm{ABAB} a c b$ & 0.243 & 0.127 \\
\hline
\end{tabular}

$b$ The uppercase and lowercase letters represent the substrate and the Pd overlayer, respectively. Fits within the error margins defined by RR are highlighted in bold with the best solutions underlined 


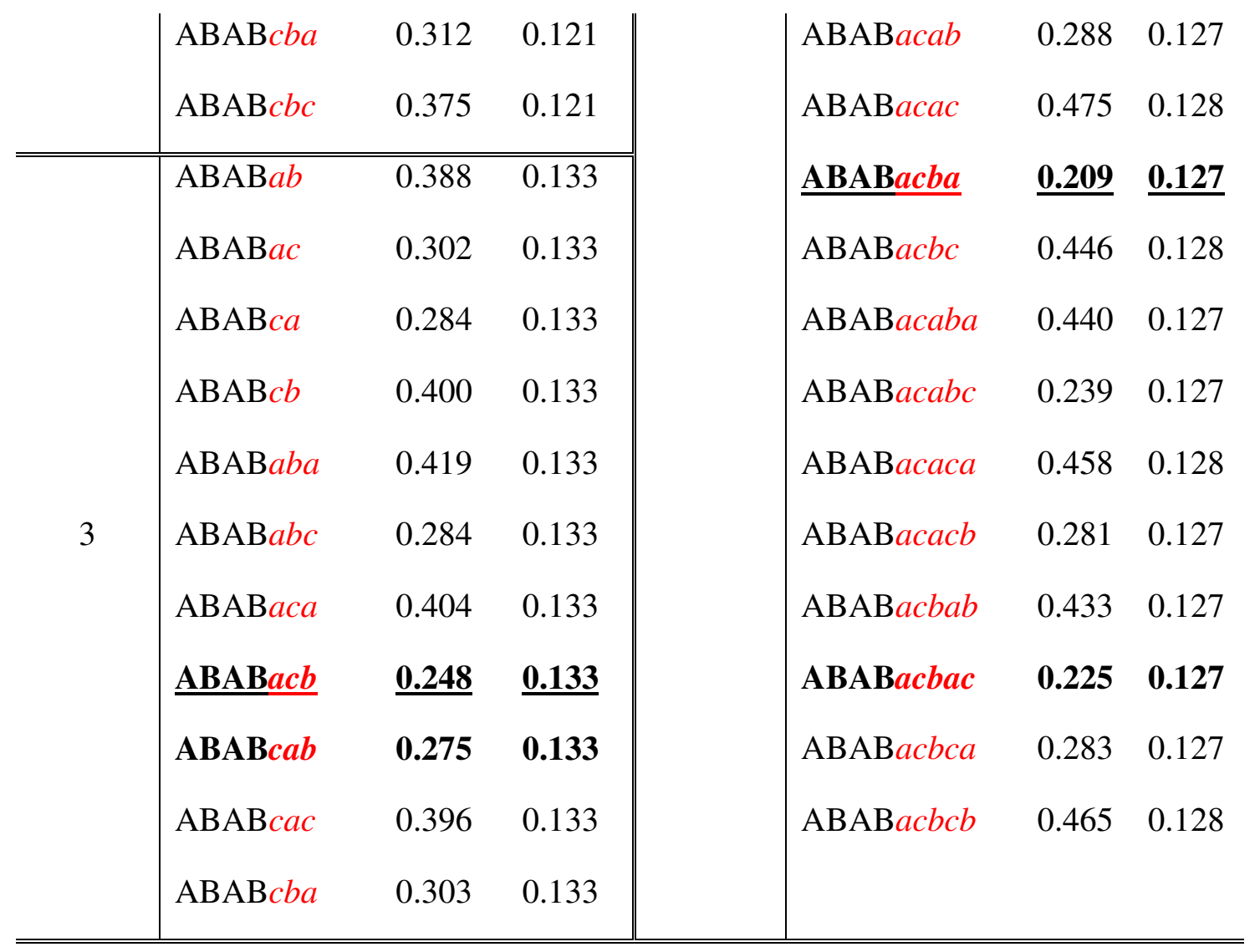

The first result to note is that the best fit structures are always for models with the same number of Pd layers as expected from the experimental deposition times. This again leads to a high confidence that the layers are growing layer by layer and that our XPS derived monolayer breakpoint analysis is correct. For 1 and $2 \mathrm{ML}$ films the models leading to $\mathrm{R}_{\mathrm{Pmin}}$ are clearly outside the statistical error bar of any other structure and represent exactly the expected film thickness. For thicker films competing models with similar $R_{P}$ can be distinguished by evaluating the upper limit $\mathrm{R}_{\mathrm{Pmax}}$ (see above). The $3 \mathrm{ML}$ models $\mathrm{ABABcab}, \mathrm{ABABabca}, \mathrm{ABABcbac}\left(\mathrm{R}_{\mathrm{P}}=\right.$ $0.275,0.272,0.270)$ are within the statistical margin of the best fit model ABABacb $\left(R_{P}=0.248\right)$ and the $4 \mathrm{ML}$ model ABABacbac $\left(\mathrm{R}_{\mathrm{P}}=0.225\right)$ competes with the best fit structure ABABacba $\left(R_{P}=0.209\right)$. All other models fall outside $R_{P \max }$ criterion. 
The best results obtained suggest a growth model in which the first layer adopts an hcp pseudomorphic structure $\mathrm{ABAB} a$; the second layer introduces a stacking fault to give a fcc like termination $\mathrm{ABAB} a c$; with subsequent layers growing in an fcc pattern to give a stacking sequence of $\mathrm{ABAB} a c b a$. This model is also consistent with what was found for the more complex epitaxially strained growth of $\mathrm{Pd}$ on $\mathrm{Ru}\{0001\}$ by LEEM/LEED on a single terrace ${ }^{28}$. The 3ML ABABcab, $\mathrm{ABAB} a b c a, \mathrm{ABAB} c b a c$ and 4ML ABABacbac structures were, however, competitive at this level with the lowest $\mathrm{R}_{\mathrm{P}}$ structures. These models were carried forward for a final refinement by optimizing the non-structural parameters: (a) Debye-Waller factor; (b) real and imaginary part of the optical potential. In doing this we found these competing trial structures resulted in $\mathrm{R}_{\mathrm{P}}$ values of 0.202 (ABABacb), 0.234 (ABABcab), 0.231 (ABABabca) and 0.232 (ABABcbac) for the 3ML models, and 0.152 (ABABacba) and 0.191 (ABABacbac) for the 4ML structures. This eliminates all but the ABABacb and ABABacba models for the $3 \mathrm{ML}$ and 4ML data, respectively. Details of these structures are listed in Table 4.

To test the possibility for in-plane relaxation into the bulk Pd structure of the thickest (4ML) films the same models were run using Pd lateral unit cell dimensions instead of Re. The resulting $\mathrm{R}_{\mathrm{P}}$ for $4 \mathrm{ML} \mathrm{ABAB} a c b a$ and $\mathrm{ABAB} a c b a c$ are 0.204 and 0.223 , respectively, which are again worse fits than for the best structures of Table 4 . We therefore conclude that the growth is predominantly layer by layer, and that the films grow to form an $\mathrm{ABAB} a c b a$ stacking sequence in single domains of fcc on each terrace with the Re in-plane lattice constant. Some example LEED-IV curves for 1-4ML thick films are shown in Figure 7. 
Table 4. Summary of LEED-IV results obtained for the interlayer spacing of 1-4 ML Pd films ${ }^{c}$ with comparison to reference [9] for 1 ML DFT results.

\begin{tabular}{|c|c|c|c|c|}
\hline No. of ML & 1 & 2 & 3 & 4 \\
\hline Structure & $\mathrm{ABAB} a$ & $\mathrm{ABAB} a c$ & $\mathrm{ABAB} a c b$ & $\mathrm{ABAB} a c b a$ \\
\hline $\mathrm{Rp}$ & 0.187 & 0.180 & 0.202 & 0.152 \\
\hline $\mathrm{RR}$ & 0.132 & 0.130 & 0.139 & 0.137 \\
\hline Energy Range & 1844.00 & 1906.25 & 1668.00 & 1705.50 \\
\hline \multirow{3}{*}{$\mathrm{d}_{12} / \AA$} & & $2.30 \pm 0.04$ & $2.34 \pm 0.04$ & $2.30 \pm 0.03$ \\
\hline & $(-1.9 \pm 2.3 \%)$ & $(+2.5 \pm 1.6 \%)$ & $(+4.3 \pm 1.9 \%)$ & $(+2.5 \pm 1.4 \%)$ \\
\hline & $2.252(+0.9 \%)^{9}$ & & & \\
\hline \multirow{3}{*}{$\mathrm{d}_{23} / \AA$} & $2.18 \pm 0.03$ & $2.23 \pm 0.03$ & $2.29 \pm 0.04$ & $2.28 \pm 0.03$ \\
\hline & $(-2 \pm 1.4 \%)$ & $(-0.5 \pm 1.6 \%)$ & $(+1.7 \pm 1.7 \%)$ & $(+1.6 \pm 1.2 \%)$ \\
\hline & $2.152(-3.5 \%)^{9}$ & & & \\
\hline \multirow{3}{*}{$\mathrm{d}_{34} / \AA$} & $2.25 \pm 0.04$ & $2.19 \pm 0.07$ & $2.28 \pm 0.07$ & $2.30 \pm 0.05$ \\
\hline & $(+0.8 \pm 1.7 \%)$ & $(-1.8 \pm 3 \%)$ & $(+1.4 \pm 3 \%)$ & $(+2.3 \pm 2.3 \%)$ \\
\hline & $2.275(+2.0 \%)^{9}$ & & & \\
\hline \multirow{3}{*}{$\mathrm{d}_{45} / \AA$} & $2.23 \pm 0.06$ & $2.30 \pm 0.07$ & $2.17 \pm 0.13$ & $2.30 \pm 0.06$ \\
\hline & $(-0.1 \pm 2.7 \%)$ & $(+3.4 \pm 3 \%)$ & $(-2.6 \pm 5.9 \%)$ & $(+2.3 \pm 2.8 \%)$ \\
\hline & bulk $^{9}$ & & & \\
\hline
\end{tabular}

${ }^{c}$ The layer spacing is both absolute distance and as a percentage of the bulk interlayer spacing, with a negative value being a contraction. The italicized entries are at the Pd-Re interface with the bulk value averaged over the $\operatorname{Pd}-\operatorname{Pd}(111)$ bulk and $\operatorname{Re}-\operatorname{Re}\{0001\}$ bulk inter-layer separations. 




Figure 7. Sample LEED-IV curves representing 1-4ML Pd deposited on $\operatorname{Re}\{0001\}$ at two angles; Normal incidence and $10^{\circ}$ off-normal. Black curves represent the experimental data and red curves represent the theoretical fits along with $R_{P}$ for each pair. The full set of LEED-IV curves are shown in Supporting Information ${ }^{26}$.

\section{Discussion}

The LEED-IV results show that the outermost Re layer tends to remain slightly contracted as Pd layers grow on top. For the 1ML film the Pd-Re layer spacing of $2.20 \pm 0.05 \AA$ compares favorably with the calculated values of $2.18 \AA$ by $\mathrm{Wu}$ and Freeman ${ }^{8}$ and $2.252 \AA$ of Pallassana $e t$ $a l .{ }^{9}$ On the clean surface and for electronegative adsorbate layers, such as S, significantly larger contractions are observed with $\mathrm{d}_{12}$ distances ranging from $2.08 \AA\left(0.25 \mathrm{ML} \mathrm{S}{ }^{33}\right)$ to $2.13 \AA$ (clean surface) or -7 to $-4 \%$. This points towards charge transfer from Pd to Re, as expected, at the interface. The 1ML data also indicates that the surface relaxation of the first Re-Re layer is not entirely lifted by the adsorption of Pd which correlates well with the theoretical work of Pallassana et al. $^{9}$.

The Pd-Pd inter-layer spacing for all multilayer films are expanded with respect to their bulk inter-layer spacing. This is a similar, but smaller, effect than that described by de Siervo et al. ${ }^{18}$ for the strained $5 \mathrm{ML} \mathrm{Pd} / \mathrm{Ru}\{0001\}$ system who suggest strain from island and domain formation is responsible for the lattice expansion. The Pd interlayer spacings of the thickest film here are, 
however, within experimental error the same as those reported by Van Hove's group for Pd(111) single crystals, which show approximately $1 \pm 2 \%$ expansion of the outermost $d_{12}$ separation $^{34}$. The origin of this expansion is the cause of some controversy in the literature as hydrogen incorporation into Pd bulk leads to a lattice expansion, so one argument is that hydrogen from residual gas in UHV could be responsible. However, it is often found theoretically that the outer layers of Pd bulk are expanded, with DFT calculations ${ }^{9}$ predicting a $d_{12}$ separation of $2.315 \AA$ $(+3.1 \%)$. Hydrogen chemisorption on the $1 \mathrm{ML} \operatorname{Pd} / \operatorname{Re}\{0001\}$ is predicted to cause large expansions of the outermost $\mathrm{d}_{12}$ separation to $2.304 \AA$ from $2.252 \AA$ for the clean film surface 9 .

The main result of the LEED-IV study is, however, to determine the stacking sequence of the film as it grows as this information cannot be reliably retrieved by scanning tunneling microscopy. As the film grows it sequentially adopts an $\mathrm{ABAB} a c b a$ stacking sequence and the fcc film grows in a single orientation dictated by the terrace upon which it nucleates. Films nucleated on the alternate terrace adopt a similar fcc stacking sequence but, due to the alternating layers in the hcp substrate, induce a stacking fault at the substrate step edge in 2 ML or greater film thicknesses. So despite a near perfect epitaxial match of nearest neighbor distances between overlayer and substrate our films are not necessarily strain and defect free. We predict that for thicker films the stacking faults induced by the substrate steps will adopt low energy configurations and may be a useful method of inducing a known defect structure into unstrained fcc bulk materials. The scanning tunneling microscopy results of Figure $5 \mathrm{c}$ and $5 \mathrm{~d}$ show this effect clearly as relatively little Pd in the second layer wets the step edges and prefers to decorate the terrace. The growth of the first Pd monolayer is also interesting as this adopts the substrate hcp structure and as such should not generate a stacking fault at the step edges. Again, however, the Pd avoids the step edges with most of the material forming fractal structures on the terrace at 
very low coverage, Figure 5a, and more compact but still dendritic structures with increased coverage, Figure 5b.

The low average coordination of submonolayer Pd at the $\operatorname{Re}\{0001\}$ surface would be expected to induce core-level shifts to lower binding energy, however, we see the opposite effect. The strong binding of $\mathrm{Pd}$ to $\mathrm{Re}$, and the fact that the $\mathrm{Pd}$ is sitting in hcp sites of the surface in preference to the usual fcc structure ${ }^{8}$, induces a very significant charge transfer from the low coordination Pd. This strong interaction broadens the surface atom d-bands ${ }^{9}$ and shifts the valence and core levels to higher binding energy in XPS. As the average coordination increases with coverage towards completing a full layer (the fractal islands fill in) the changes in local environment move the peak towards the bulk position. Structural relaxations have also been noted to cause small corrections to binding energy shifts predicted on the basis of coordination in experiment $^{35}$ and theory ${ }^{11}$. The LEED-IV from multilayer films indicate the initial Pd contraction relaxes into an expansion and so may be expected to yield a shift of the states to lower binding energy. Eventually we have a three layer system with a wide d-band positive core-level shifted Pd layer at the interface buried beneath a bulk like Pd with an outer surface which is expected to show a negative binding energy shift due to under-coordination and slightly narrower bands. The low resolution XPS data however preclude detailed examination of the layer by layer core level shifts but both the magnitude and sign are comparable to the existing literature. Our shift occurs over a much narrower coverage range than hitherto reported (2ML here versus 5-7 ML in refs [2] and [5] respectively) as would be expected for a chemical bonding effect localized at the interface. We conclude that the strong interaction of $\mathrm{Pd}$ and $\mathrm{Re}$ lead to chemical ligand effects that out compete the opposing shifts induced by low coordination. These effects diminish with coverage by $2 \mathrm{ML}$ as the second monolayer covers the first. 


\section{Conclusion}

The $\operatorname{Re}\{0001\}$ surface has been employed as a substrate for the unstrained epitaxial growth of Pd ultra-thin films. The use of multiple angle simultaneous LEED-IV structural refinement enabled reliable surface structure determination in the presence of two terrace types. The growth of 1-4 ML Pd thin films on the Re $\{0001\}$ surface was investigated by LEED-IV, XPS, and STM. LEED-IV showed the film to follow $\mathrm{ABAB} a c b a$ stacking sequence with thicker layers expanding with respect to Pd bulk inter-layer spacing. 1 and 2 ML films display a contraction at the Pd-Re interface due to the strong chemical interaction of the metals. The trend in the core level shifts towards a lower binding energy with increasing coverage agrees with the experimental literature, however, our shift occurs over a much narrower coverage range than reported hitherto. The out of plane contraction of the films is reduced with increasing thickness, and the outer layers expand with respect to bulk Pd values. STM images of submonolayer coverage show fractal islands that do not cover any steps at $0.25 \mathrm{ML}$ which change to more compact islands at $0.4 \mathrm{ML}$. At $1.25 \mathrm{ML}$ the islands formed are of hexagonal shapes with at least $93 \%$ of the surface covered before the second layer starts to grow. Step edges show different structures for the $1 \mathrm{ML}$ Pd and the $2 \mathrm{ML}$ Pd, the former being much more irregular than the close packed structures of the latter. The structural data, stacking sequence, and correlation of the core level shifts with well characterized film thickness will inform theoretical and experimental work on the heteroepitaxial growth of $\mathrm{Pd}$, and for Pd coated nanostructured catalysts. We hope to make detailed measurements of the core-level fine structure in future.

\section{Acknowledgment}

We thank Grongar Lloyd and Richard Palmer at the University of Birmingham, UK for the permanent loan of the STM. H.A.E. and Z.V.Z. acknowledge the EPSRC and the "Research 
Endowment Trust Fund" of the University of Reading for postgraduate studentships, respectively. We thank the Royal Society / Wolfson Foundation for laboratory refurbishment grant and Alan Adams for engineering the sample transfer system.

\section{Supporting Information Available}

This information is available free of charge via the Internet at http://pubs.acs.org.

\section{References}

1 Hwang, R. Q.; Bartelt, M. C. Chem. Rev. 1997, 97, 1063

2 Mun, B. S.; Lee, C.; Stamenkovic, V.; Markovic, N. M.; Ross Jr, P. N. Phys. Rev. B 2005, 71,115420

3 Greeley, J.; Mavrikakis, M. Catal. Today 2006, 111, 52.

4 Santos, B.; Puerta, J. M.; Cerda, J. I.; Herranz, T.; McCarty, K. F.; de la Figuera, J.; New J. Phys. 2010, 12, 023023

5 Campbell, R. A.; Rodriguez, J. A.; Goodman, D. W. Phys. Rev. B 1992, 46, 7077

6 Pick, S. J. Phys. Chem. 1995, 99, 15375

7 Golfetto, E.; Baraldi, A.; Pozzo, M.; Alfê, D.; Lacovig, P.; Veselli, E.; Lizzzit, S.; Comelli, G.; Rosei, R. J. Phys. Chem. C 2010, 114, 436.

8 Wu, R.; Freeman, A. J. Phys. Rev. B 1995, 52, 12419

9 Pallassana, V.; Neurock, M.; Hansen, L. B.; Hammer, B.; Nørskov, J. K. Phys. Rev. B 1999, 60,6146 
10 Hammer, B.; Morikawa, Y.; Nørskov, J. k. Phys. Rev. Lett. 1996, 76, 2141

11 Ruban, A.; Hammer, B.; Stoltze, P.; Skriver, H. L.; Nørskov, J. K. J. Mol. Catal. A: Chem. 1997, $115,421$.

12 Andersen, J. N.; Hennig, D.; Lundgren E.; Methfessel M.; Nyholm R. and Scheffler M., Phys. Rev. B 1994, 50,17525

13 Bianchettin, L.; Baraldi, A.; de Gironcoli, S.; Vesselli, E.; Lizzit, S.; Petaccia, L.; Comelli, G.; Rosei, R. J. Chem. Phys. 2008, 128, 114706

14 Vesselli, E.; Baraldi, A.; Bondino, F.; Comelli, G.; Peressi, M.; Rosei, R. Phys. Rev. B 2004, 70,115404

15 Oba, Y; Sato, T.; Shinohar, T. Phys. Rev. B 2008, 78, 224417

16 Hüger, E.; Osuch, K. Europhys. Lett. 2003, 63, 90

17 Alexandre, S. S.; Anglada, E.; Soler, J. M.; Yndurain, F. Phys. Rev. B 2006, 74, 054405

18 de Siervo, A.; De Biasi, E.; Garcia1, F.; Landers, R.; Martins, M. D.; Macedo, W. A. A. Phys. Rev. B 2007, 76, 075432

19 McCavish, N. D.; Bennett, R.A Surf. Sci. 2003, 546, 47

20 Bennett, R. A.; Mulley, J. S.; Newton, M. A.; Surman, M. J. Chem. Phys. 2007, 127, 1

21 Shih, H. D.; Jona, F.; Jepsen, D. W.; Marcus, P. M. J. Phys. C: Solid State Phys. 1976, 9, 1405

22 http://www.reading.ac.uk/web/FILES/chemistry/Georg_Held_LEED_Description.pdf 
23 Held, G.; Braun, W. CLEED manual, available from the authors

24 Pendry, J. B. Low Energy Electron Diffraction: Academic Press: London, 1974.

25 Van Hove M. A.; Tong, S. Y. Surface Crystallography by LEED: Springer: Berlin, 1979.

26 Supporting Information.

27 Pendry, J. B. J. Phys. 1980, C 13, 937

28 de la Figuera, J.; Puerta, J. M.; Cerda, J.I.; El Gabaly, F.; McCarty, K. F. Surf. Sci. 2006, $600, \mathrm{~L} 105$

29 Somorjai, G. A. Introduction to surface chemistry and catalysis: Wiley: New York, 1994

30 http://www.casaxps.com/

31 Witten Jr., T. A.; Sander, L. M. Phys. Rev. Lett. 1981, 47, 1400

32 Röder, H.; Bromann, K.; Brune, H.; Kern, K. Phys. Rev. Lett. 1995, 74, 3217

33 Barbieri, A; Jentz D.; Materer N.; Held, G.; Dunphy J.; Ogletree D.F.; Sautet P.; Salmeron M.; Van Hove, M.A.; and Somorjai, G.A. Surf. Sci. 1994, 312, 10

34 Felter, T. E.; Sowa, E. C.; Van Hove, M. A. Phys. Rev. B: Cond. Matt. 1989, 40, 891

35 Baraldi, A.; Bianchettin, L.; Vesselli, E.; de Gironcoli, S.; Lizzit, S.; Petaccia, L.; Zampieri, G.; Comelli, G. and Rosei, R. New J. Phys. 2007, 9, 143. 
TOC

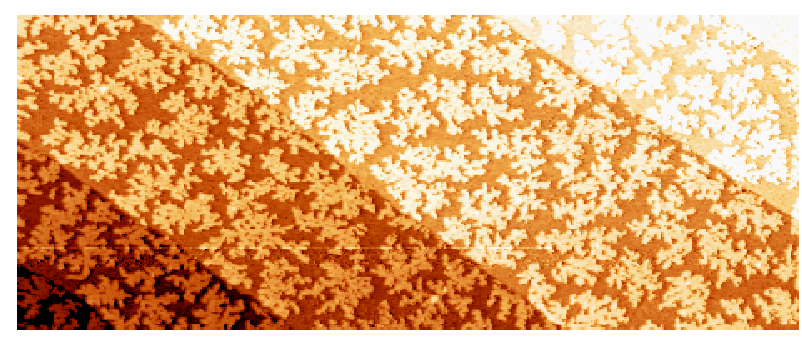

